\title{
VULNERABILIDADE NATURAL E PERIGO DE CONTAMINAÇÃO DO SISTEMA AQUÍFERO DUNAS NA REGIÃO NORTE DO MUNICÍPIO DE AQUIRAZ, CEARÁ
}

\author{
Karen Vendramini de ARAÚJO \\ Itabaraci Nazareno CAVALCANTE \\ Rafael Mota de OLIVEIRA \\ Filipe da Silva PEIXOTO \\ Inácio Ocinaí de LIMA NETO
}

\begin{abstract}
RESUMO
Este trabalho apresenta o estudo da vulnerabilidade natural e perigo de contaminação do Sistema Aquífero Dunas na região norte do município de Aquiraz (CE), utilizando o método GOD ( $\mathrm{G}$ - grau de confinamento; $\mathrm{O}$ - estratos de cobertura da zona saturada; D - profundidade do lençol freático), associado ao potencial contaminante do sistema de saneamento in situ, que utiliza fossas como meio de deposição de efluentes domésticos. Foram analisados dados geológicos e hidrogeológicos de poços tubulares obtidos por meio do banco de dados SIAGAS e durante etapa de campo. Na área de estudo, o Sistema Aquífero Dunas é constituído por areias eólicas e apresenta nível estático entre 2,0 e 61,0 m de profundidade, com predominância de valores até $20,0 \mathrm{~m}$. A vulnerabilidade GOD variou de média a alta, com vulnerabilidade média em $76 \%$ da área. O potencial contaminante das atividades antrópicas na área foi estimado por meio da análise da densidade de fossas, tendo em vista que estes sistemas estáticos são considerados de alto potencial à contaminação, decorrente da aplicação constante de carga contaminante no subsolo. A avaliação do perigo de contaminação do Sistema Aquífero Dunas foi realizada com base na comparação das condições naturais com as atividades antrópicas. Verificou-se que as áreas com maior perigo de contaminação são aquelas que apresentam a maior densidade de fossas, associada aos elevados índices de vulnerabilidade GOD encontrados na área. Espera-se que os resultados apresentados possam se constituir em subsídios para a gestão dos recursos hídricos subterrâneos da região.
\end{abstract}

Palavras-chave: Aquiraz; Hidrogeologia; Sistema Aquífero Dunas; Vulnerabilidade; GOD; Perigo de contaminação.

\section{ABSTRACT}

NATURAL VULNERABILITY AND CONTAMINATION HAZARD OF THE DUNAS AQUIFER SYSTEM IN THE NORTHERN REGION OF THE MUNICIPALITY OF AQUIRAZ, CEARÁ. This paper examines the natural vulnerability and contamination hazard of the Dunas Aquifer System in the northern region of the municipality of Aquiraz (CE), using the GOD (G - groundwater hydraulic confinement, $\mathrm{O}$ - overlying strata/lithology, D - depth to water table) method and taking into account potential contamination by the in situ sanitation system, which uses pits as a means of depositing domestic effluents. Geological and hydrogeological data were analyzed from tubular wells obtained through the SIAGAS database and during the field work. The Dunas Aquifer System in the study area consists of eolian sands and presents static levels between 2.0 and $61.0 \mathrm{~m}$ depth, with a predominance of values less than $20.0 \mathrm{~m}$. The GOD vulnerability varied from medium to high, with 
average vulnerability in most of the area $(76 \%)$. The potential contamination by the anthropic activities in the area was estimated by means of the analysis of the density of cesspools, given that these static systems have a high contamination potential because of the constant application of contaminant load in the subsoil. Assessment of the contamination hazard of the Dunas Aquifer System was based on the comparison of the natural conditions and the anthropic activities. It was found that the areas with the highest risk of contamination are those with the highest density of cesspools, associated with the highest GOD vulnerability indexes. These results will be useful for managment of groundwater resources in the region.

Keywords: Aquiraz; Hydrogeology; Dunas Aquifer System; Vulnerability; GOD; Contamination hazard.

\section{INTRODUÇÃO}

A explotação excessiva dos recursos hídricos subterrâneos, a ocupação irregular do solo e a ausência de utilização apropriada dos instrumentos legais existentes colocam em risco a qualidade das águas subterrâneas. A ocupação urbana desordenada sem planejamento no que se refere a infraestrutura, saneamento básico e coleta de resíduos, representa um dos principais fatores que contribuem para o comprometimento da qualidade dos recursos hídricos.

Tendo em vista a conservação da água subterrânea, faz-se necessária a implementação de medidas de controle do uso do solo. Atividades relacionadas à implantação de loteamentos e à expansão dos núcleos urbanos devem levar em consideração a proteção de áreas destinadas ao uso racional do solo (LOPES et al. 2012).

A região norte do município de Aquiraz (CE) possui intensa atividade turística, com demanda hídrica crescente; em contrapartida, não é atendida por rede de água e esgoto. $\mathrm{O}$ abastecimento é realizado exclusivamente por água subterrânea, tendo com fonte principal o Sistema Aquífero Dunas. Este sistema constitui-se de aquíferos livres formados por sedimentos quaternários, principalmente areias quartzosas de granulação fina a média, bem selecionadas, com intercalações ocasionais de níveis silto-argilosos a argilosos (MOURA et al. 2016). O caráter livre deste sistema aquífero torna-o mais suscetível à contaminação proveniente de atividades antrópicas.

Devido à ausência de rede de esgoto na região, a população depende de sistemas de saneamento in situ, que utiliza fossas ou latrinas. Este sistema, segundo FOSTER et al. (2006), funciona por percolação do líquido efluente no solo, e, em perfis de solo permeáveis, isso resulta em infiltra- ção que alcança o aquífero. As partes sólidas, que deveriam ser periodicamente removidas e destinadas a outro local, em muitos casos permanecem no solo e são progressivamente lixiviadas pela água da chuva ou por outros fluidos que se infiltram. Mesmo quando bem construídas e operadas, as fossas geram cargas de compostos de nitrogênio (inicialmente na forma de amônio, mas em geral na forma de nitrato, mais estável) capazes de contaminar os aquíferos.

Segundo HIRATA \& FERREIRA (2001), a avaliação da vulnerabilidade apresenta-se como instrumento útil para a preservação da qualidade das águas subterrâneas, de forma a orientar as áreas mais adequadas e menos suscetíveis para a instalação e desenvolvimento de atividades potencialmente poluidoras.

O termo vulnerabilidade à contaminação do aquífero é usado para representar as características intrínsecas que determinam a suscetibilidade de um aquífero a ser adversamente afetado por uma carga contaminante (FOSTER \& HIRATA 1988).

Diversos métodos foram desenvolvidos desde que os primeiros estudos de vulnerabilidade foram apresentados, sendo os métodos DRASTIC (ALLER et al. 1987) e GOD (FOSTER \& HIRATA 1988) comumente utilizados no Brasil.

Neste trabalho optou-se pela utilização do método GOD ( $\mathrm{G}$ - grau de confinamento; O - estratos de cobertura da zona saturada; D - profundidade do lençol freático), dada a sua maior simplicidade de aplicação, em decorrência da utilização de parâmetros geralmente disponíveis ou facilmente determinados.

O método GOD (FOSTER \& HIRATA 1988) é baseado na avaliação do nível de acessibilidade hidráulica da zona saturada, em função do grau de confinamento do aquífero, da profundidade do ní- 
vel estático e da capacidade de atenuação dos contaminantes ao longo da zona vadosa.

MOURA et al. (2016) aplicaram o método GOD para avaliação da vulnerabilidade do Sistema Aquífero Dunas no complexo industrial e portuário do Pecém no estado do Ceará. Os resultados mostraram a predominância de vulnerabilidade alta, em função do baixo grau de consolidação da zona vadosa formada predominantemente por areias eólicas.

CUTRIM \& CAMPOS (2010) salientam que a aplicação do método GOD é especialmente importante em áreas urbanas, em que grande parte do abastecimento é feito por água subterrânea com risco efetivo de contaminação, cenários que necessitam do conhecimento da vulnerabilidade de aquífero para proposição de medidas preventivas e corretivas apropriadas.

O perigo de contaminação de um aquífero num dado local pode ser determinado considerando-se a interação entre a carga contaminante aplicada ao subsolo como resultado de atividades humanas e a vulnerabilidade do aquífero à contaminação, o que depende das características naturais dos estratos que o separam da superfície do solo (FOSTER et al. 2006).

PEREIRA JÚNIOR et al. (2015) avaliaram o risco de contaminação do Aquífero Bauru no município de Rio Verde, estado de Goiás. Estes autores realizaram modificações na metodologia proposta por FOSTER et al. (2006), sobretudo, em fontes pontuais; para estes casos foram definidos empiricamente raios de influência na tentativa de melhor representar a área de influência destes pontos. A determinação do risco se deu a partir do cruzamento dos dados oriundos das fontes de poluição e da vulnerabilidade GOD.

$\mathrm{Na}$ área de estudo, o perigo à contaminação da água subterrânea está associado, sobretudo, a fossas como meio de deposição e tratamento de efluentes domésticos. Devido a intensa atividade turística na área, a população flutuante sazonal proporciona um acréscimo considerável à população local, aumentando de maneira significativa a quantidade de efluentes domésticos.

O presente estudo buscou avaliar a vulnerabilidade natural e o perigo de contaminação do Sistema Aquífero Dunas, tendo em vista a sua importância como fonte de abastecimento local, visando fornecer subsídios para o planejamento urbano e conservação das águas subterrâneas em áreas turísticas, com grande potencial de uso desse recurso.

\section{2 ÁREA DE ESTUDO}

A área de estudo está localizada na porção norte do município de Aquiraz, cerca de $30 \mathrm{~km}$ à leste da cidade de Fortaleza (CE) e corresponde à praia do Porto das Dunas com $9 \mathrm{~km}^{2}$ (Figura 1). O acesso principal, partindo de Fortaleza, é realizado por meio das rodovias CE-040 e CE-025.

De acordo com o censo demográfico de 2010 realizado pelo IBGE, o município de Aquiraz possui $482,573 \mathrm{~km}^{2}$ e 72.628 habitantes, divididos em população urbana com 67.083 habitantes e rural com 5.545 habitantes, refletindo uma densidade demográfica de 150,5 hab. $/ \mathrm{km}^{2}$ a uma taxa de crescimento anual de $1,85 \%$ e uma taxa de urbanização de $92,37 \%$.

O abastecimento de água do município possui uma taxa de cobertura de $49,44 \%$; o esgotamento sanitário possui uma cobertura de $22,79 \%$ e o fornecimento de energia elétrica abrange $99,10 \%$ das residências (IPECE 2012).

O município de Aquiraz possui um turismo intenso, principalmente na região do Porto das Dunas onde existem diversos empreendimentos hoteleiros e recreativos, constituindo a principal atividade econômica da área.

Essa região, ao longo do tempo e em toda sua extensão, vem sendo ocupada de forma irregular desde a década de 1980, principalmente em alguns pontos onde a atividade turística encontra-se concentrada (CÂMARA et al. 2010).

De acordo com a Resolução CONAMA $n^{\circ}$ 341/03 (BRASIL 2003), as dunas desprovidas de vegetação somente poderão ser ocupadas com atividade ou empreendimento turístico sustentável em até $20 \%$ de sua extensão, limitada à ocupação a $10 \%$ do campo de dunas, recobertas ou desprovidas de vegetação.

A ocupação no Porto das Dunas chega a 80\%, sendo caracterizada, principalmente, por empreendimentos turísticos e casas de veraneio, além de imóveis residenciais, comerciais e alguns sítios (Figura 2).

\subsection{Aspectos geoambientais}

O município apresenta um regime de chuvas tropicais com alternância de episódios secos e úmidos ao longo do ano. O período de maior precipitação ocorre nos meses de janeiro a junho. Nos demais meses, as precipitações são escassas, com chuvas irregulares decorrentes das linhas de instabilidade geradas pelo efeito da brisa marítima. 




LEGENDA

$\square$ Área de estudo

- Rio Pacoti

- Rodovia CE-025

Poços cadastrados

* Usina eólica da Prainha

Limite municipal

Estuário do rio Pacoti

Qa Sistema Aquifero Aluvionar

ad Sistema Aquífero Dunas

Tb Sistema Aquifero Barreiras

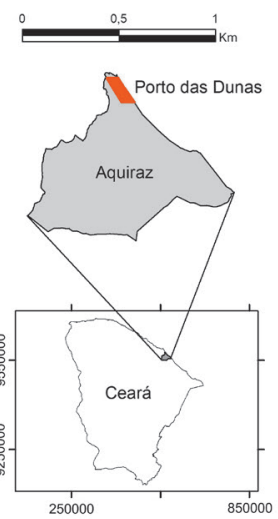

Projeçáo Cartográfica UTM
Datum SIRGAS 2000, 24 S

FIGURA 1 - Localização da área de estudo e distribuição dos poços cadastrados no Sistema Aquífero Dunas.

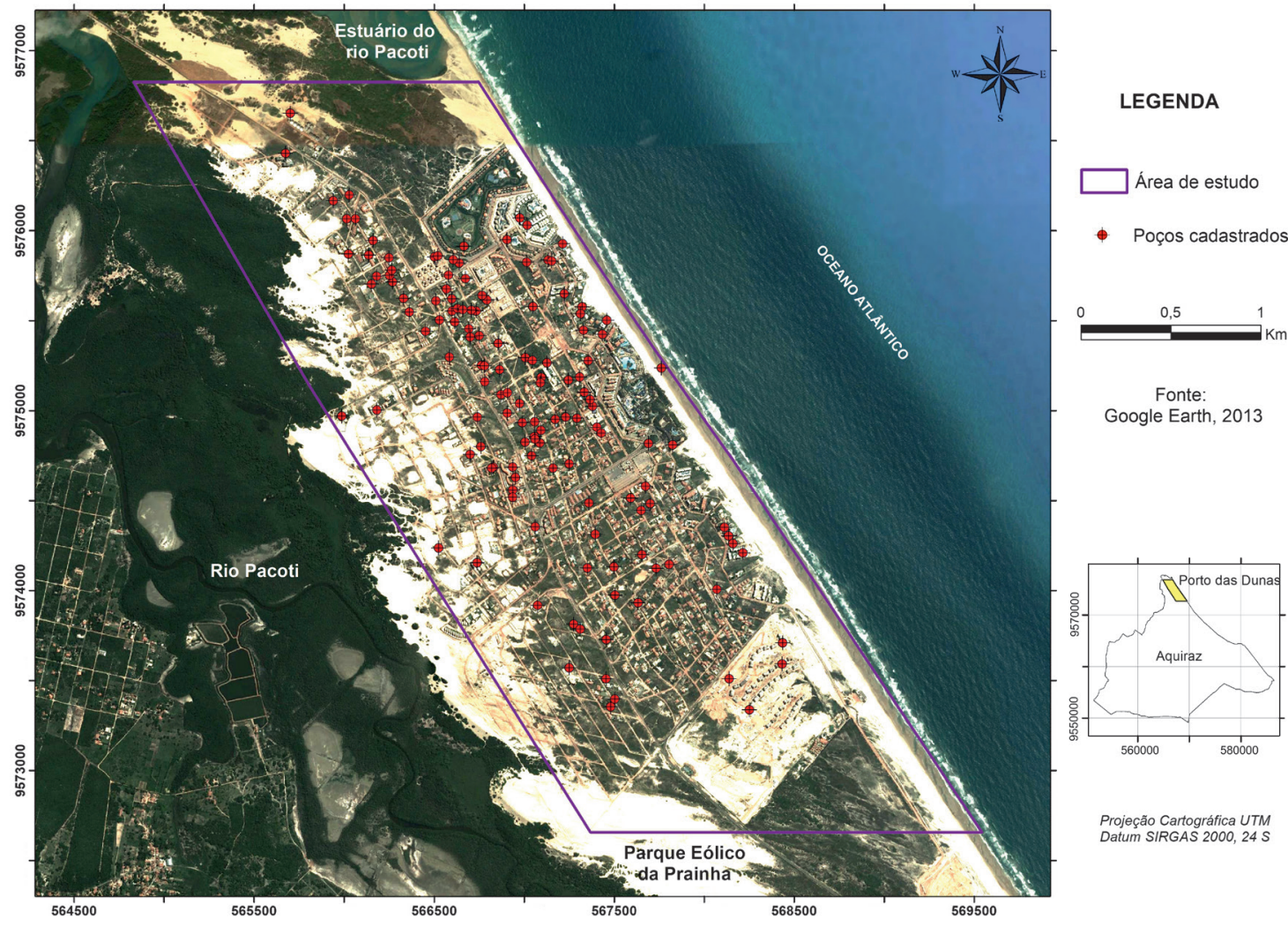

FIGURA 2 - Ocupação urbana no Porto das Dunas, Aquiraz/CE. 
A área de pesquisa está inserida no domínio geomorfológico da Planície Litorânea, composto por campos de dunas e praias. Na região se desenvolvem, predominantemente, os neossolos quartzarênicos marinhos, caracterizados como solos de baixa fertilidade, excessivamente drenados, apresentando sequência de horizontes A e C. Em geral o horizonte A, fracamente desenvolvido, apresenta textura arenosa e coloração cinza escura a muito escura. $\mathrm{O}$ horizonte $\mathrm{C}$, com características semelhantes ao A, exibe coloração mais clara, geralmente cinza clara a bruno amarelado (BRANDÃO 1995).

A geologia é caracterizada pela presença de sedimentos quaternários representados por depósitos eólicos, que correspondem a dunas constituídas por areias esbranquiçadas, indicando ausência de ferro, com granulometria variando de fina a média. A composição mineralógica é principalmente quartzo e minerais pesados (FREIRE 1989).

Incluídas no contexto estratigráfico das dunas móveis, as praias recentes são constituídas por areias quartzosas não consolidadas, de granulometria média a grossa, depositadas ao longo da costa, numa faixa que compreende desde a linha de maré baixa até a base das dunas móveis.

\subsection{Hidrogeologia}

O Sistema Aquífero Dunas engloba tanto dunas fixas quanto dunas móveis, devido à semelhança nas características litológicas e hidrodinâmicas de ambas. Segundo CAVALCANTE (1998), esse sistema possui dupla função hidrogeológica, uma como aquífero principal e outra como aquífero de transferência para os aquíferos sotopostos, como por exemplo o Aquífero Barreiras.

Na zona costeira do estado do Ceará, o Sistema Aquífero Dunas apresenta valores médios de condutividade hidráulica e transmissividade que oscilam de 0,73 a $2,50 \times 10^{-4} \mathrm{~m} \mathrm{~s}^{-1}$ e de 2,37 a 6,98 $\mathrm{m}^{2} \mathrm{~h}^{-1}$, respectivamente (CAVALCANTE 1998).

Porém, é bastante suscetível à poluição, tornando-se vulnerável aos impactos causados pelo uso e ocupação do solo (MELO \& FIGUEREDO 1990, MOURA et al. 2016). A recarga ocorre principalmente através de infiltração pluvial direta e os exutórios são o Oceano Atlântico, a evapotranspiração, associada a um nível estático subaflorante, especialmente nas zonas de menores cotas altimétricas, e a transferência de água para o Aquífero Barreiras sotoposto. Os poços tubulares também representam saídas importantes, pela extração de água para diversos fins.

\section{MATERIAIS E MÉTODOS}

A caracterização hidrogeológica da área de estudo foi feita com base no Mapa Geológico do Estado do Ceará (CAVALCANTE et al. 2003) e na interpretação dos perfis construtivos-litológicos de 10 poços tubulares identificados na área.

O levantamento de 139 poços tubulares foi realizado por meio da consulta ao banco de dados do Sistema de Informações de Água SubterrâneaSIAGAS (CPRM 2013). Os dados de nível estático (NE) foram obtidos a partir do mesmo cadastro e de medições realizadas em campo.

Para a avaliação da vulnerabilidade foi utilizada a metodologia GOD (FOSTER \& HIRATA 1988). Esse método é um dos mais utilizados, principalmente por demandar uma menor gama de parâmetros, o que resulta em maior rapidez e menor custo para sua obtenção. Além disso, mostra-se mais adequado à área, tendo em vista que o Sistema Aquífero Dunas configura um meio homogêneo e isotrópico, sem grande variabilidade em relação a tipos de solo, natureza do substrato geológico, recarga e condutividade hidráulica.

Apesar da utilização mais generalizada do método DRASTIC, que considera os parâmetros: profundidade da zona sub-saturada, recarga, meio aquífero, solo, topografia, material da zona subsaturada e condutividade hidráulica, este método possui maiores dificuldades de aplicação em função dos pesos definidos arbitrariamente (KUMAR et al. 2015).

Diversos estudos buscam comparar a eficiência dos métodos DRASTIC e GOD (PONTES et al. 2009, BOUFEKANE \& SAIGHI 2013, REGINATO \& AHLERT 2013, ROSENBERGER et al. 2013, MOURA 2016), na maioria dos casos, os autores apontam que os dois métodos são satisfatórios na avaliação da vulnerabilidade de aquíferos, embora não definam qual o mais adequado.

A metodologia GOD envolve os seguintes estágios: identificação do grau de confinamento de água subterrânea $(G)$; especificação dos estratos de cobertura da zona saturada do aquífero em termos de grau de consolidação $(O)$ e estimativa da profundidade até o lençol freático (para aquíferos não confinados) ou da profundidade do primeiro nível principal de água subterrânea (para aquíferos confinados) $(D)$. O índice final de vulnerabilidade do aquífero é o produto dos índices desses parâmetros (Figura 3). 
GRAU DE CONFINAMENTO

DA ÁGUA SUBTERRÂNEA

\section{Ocorrência de ESTRATOS DE COBERTURA}

(característica litológica e grau de consolidação da zona vadosa ou camada confinante)

\section{$(x)$ \\ DistânCIA ATÉ O Lençol FREÁTICO OU O TETO DO AQÜÍFERO CONFINADO}

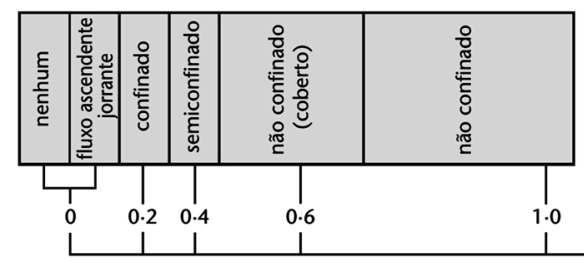

solos silte, loess, areia areia aluvial cascalho de NÃO CONSOLIDADA estuarina residuais till glacial eólica e fluvioglacial leques aluviais $\quad$ (sedimentos)

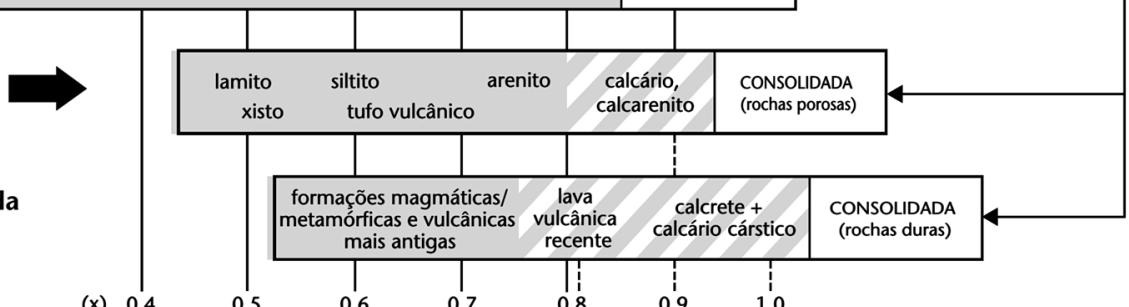

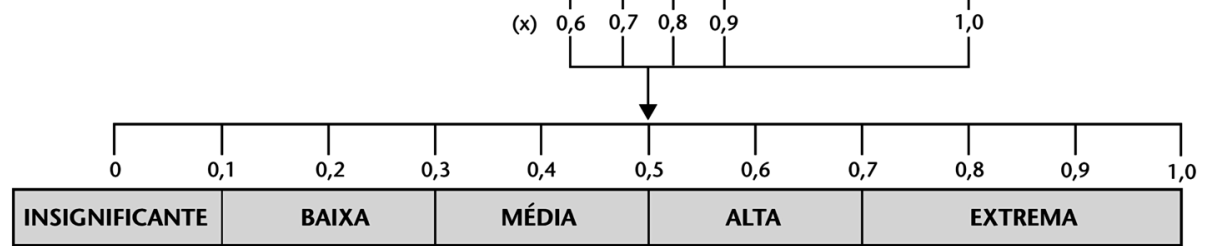

VULNERABILIDADE DO AQUÚFERO À CONTAMINAÇÃO

FIGURA 3 - Método GOD para determinação da vulnerabilidade do aquífero à contaminação (Extraído de FOSTER et al. 2006).

Neste trabalho, atribuiu-se índice 1,0 para o grau de confinamento $(G)$, dado o caráter não confinado do Sistema Aquífero Dunas.

Com base na constituição litológica, composta por sedimentos arenosos inconsolidados de origem eólica, foi atribuído índice 0,6 para o parâmetro $(O)$, ocorrência dos estratos de cobertura.

A profundidade do nível estático $(D)$ foi estabelecida com base em 22 poços distribuídos na área de estudo.

Para a elaboração do mapa de vulnerabilidade, os índices obtidos foram tratados em ambiente SIG (Sistema de Informações Geográficas). Mapas em formato raster dos parâmetros $\mathrm{O}$ e $\mathrm{D}$ foram multiplicados para a obtenção da vulnerabilidade pelo método GOD.

A avaliação do potencial à contaminação foi realizada com base na densidade das fossas das residências e/ou hotéis. O levantamento destas fontes foi feito durante etapa de campo realizada em agosto/2013, na qual foram identificadas 138 fossas.

O mapa de densidade de fossas foi elaborado utilizando o estimador de Kernel que, segundo SILVERMAN (1986), desenha uma vizinhança circular ao redor de cada ponto da amostra, correspondendo ao raio de influência, e então é aplicada uma função matemática de 1 , na posição do ponto, a 0 , na fronteira da vizinhança. $\mathrm{O}$ valor para a célula é a soma dos valores Kernel sobrepostos, e divididos pela área de cada raio de pesquisa.

Foi atribuído o valor de $250 \mathrm{~m}$ para o raio de influência que baseou a análise espacial de Kernel. Este foi determinado em função da melhor representatividade zonal da densidade de fossas na área, vinculando assim, esse fenômeno ao seu potencial contaminante. A partir daí foram definidas zonas de alta, média e baixa densidade de fossas. 
O perigo de contaminação das águas subterrâneas foi avaliado a partir do cruzamento dos dados de vulnerabilidade e da densidade de fossas, por meio do emprego da matriz de correlação (Tabela 1) em ambiente SIG.

TABELA 1 - Classificação do perigo de contaminação da água subterrânea por fontes potenciais.

\begin{tabular}{|c|c|c|c|c|}
\hline & \multicolumn{3}{|c|}{$\begin{array}{c}\text { Densidade de fossas } \\
\text { (carga de contaminação) }\end{array}$} \\
\hline & & Baixa & Média & Alta \\
\hline \multirow{2}{*}{ 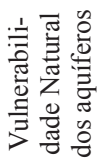 } & Média & Médio & Médio & Alto \\
\hline & Alta & Médio & Alto & Alto \\
\hline
\end{tabular}

\section{RESULTADOS E DISCUSSÃO}

A profundidade do nível estático obtida em 22 poços apresentou valores variando entre 2,0 e $61,0 \mathrm{~m}$ de profundidade, com predominância de valores até $20,0 \mathrm{~m}$. A área de estudo apresenta nível estático raso em toda a faixa leste próxima ao mar, com profundidade até $15,0 \mathrm{~m}$, e nível estático mais profundo na porção oeste, cujas profundidades são superiores a 30,0 m (Figura 4, Tabela 2).

TABELA 2 - Poços cadastrados no Porto das Dunas, Aquiraz-CE.

\begin{tabular}{lcccr}
\hline & \multicolumn{2}{c}{ Coordenadas } & $\begin{array}{c}\text { Prof.do } \\
\text { poço } \\
\text { Poço }\end{array}$ & $\begin{array}{c}\text { Nivel } \\
\text { estático } \\
(\mathrm{m})\end{array}$ \\
\cline { 2 - 4 } & UTM $(N)$ & UTM $(E)$ & 84,0 & 2,0 \\
P01 & 9576430 & 565675 & 8,0 & 25,0 \\
P02 & 9575946 & 566161 & 48,0 & 32,0 \\
P03 & 9575554 & 566600 & 60,0 & 4,5 \\
P04 & 9575576 & 567323 & 27,5 & 3,6 \\
P05 & 9576030 & 567018 & 22,5 & 2,8 \\
P06 & 9576071 & 566977 & 24,0 & 3,4 \\
P07 & 9574351 & 568114 & 26,0 & 3,4 \\
P08 & 9574304 & 568136 & 26,5 & 22,2 \\
P09 & 9576653 & 565700 & 52,0 & 3,0 \\
P10 & 9575820 & 566640 & 20,0 & 2,0 \\
P11 & 9575854 & 566503 & 40,0 & 25,0 \\
P12 & 9575860 & 566520 & 60,0 & 7,5 \\
P13 & 9574705 & 567250 & 12,0 & 8,5 \\
P14 & 9574261 & 568160 & & - \\
P15 & 9574210 & 568216 & - & 6,3 \\
P16 & 9573338 & 568252 & 8,0 & 7,0 \\
P17 & 9573510 & 568139 & 9,0 & 7,9 \\
P18 & 9575004 & 566182 & 90,0 & 52,5 \\
P19 & 9574146 & 567806 & 10,0 & 4,1 \\
P20 & 9575266 & 567127 & & 7,1 \\
P21 & 9575747 & 566181 & 80,0 & 40,0 \\
P22 & 9574236 & 566525 & 96,0 & 61,0 \\
\hline & & & &
\end{tabular}

Com base nos índices calculados, o Sistema Aquífero Dunas se enquadra em duas classes de vulnerabilidade: média $(0,3$ a 0,5$)$ e alta $(0,5$ a $0,7)$ (Tabela 3). A vulnerabilidade média ocorre em grande parte da área de pesquisa, porém, a porção leste, próxima à linha de costa, apresenta alta vulnerabilidade (Figura 5).

TABELA 3 - Índice de vulnerabilidade GOD para o sistema aquífero Dunas.

\begin{tabular}{lcccc}
\hline Poço & Confinamento & Litologia & $\begin{array}{c}\text { Nivel } \\
\text { estático }\end{array}$ & GOD \\
\hline P01 & 1,0 & 0,6 & 0,9 & 0,54 \\
P02 & 1,0 & 0,6 & 0,7 & 0,42 \\
P03 & 1,0 & 0,6 & 0,7 & 0,42 \\
P04 & 1,0 & 0,6 & 0,9 & 0,54 \\
P05 & 1,0 & 0,6 & 0,9 & 0,54 \\
P06 & 1,0 & 0,6 & 0,9 & 0,54 \\
P07 & 1,0 & 0,6 & 0,9 & 0,54 \\
P08 & 1,0 & 0,6 & 0,9 & 0,54 \\
P09 & 1,0 & 0,6 & 0,7 & 0,42 \\
P10 & 1,0 & 0,6 & 0,9 & 0,54 \\
P11 & 1,0 & 0,6 & 0,9 & 0,54 \\
P12 & 1,0 & 0,6 & 0,7 & 0,42 \\
P13 & 1,0 & 0,6 & 0,8 & 0,48 \\
P14 & 1,0 & 0,6 & 0,8 & 0,48 \\
P15 & 1,0 & 0,6 & 0,8 & 0,48 \\
P16 & 1,0 & 0,6 & 0,8 & 0,48 \\
P17 & 1,0 & 0,6 & 0,8 & 0,48 \\
P18 & 1,0 & 0,6 & 0,6 & 0,36 \\
P19 & 1,0 & 0,6 & 0,9 & 0,54 \\
P20 & 1,0 & 0,6 & 0,8 & 0,48 \\
P21 & 1,0 & 0,6 & 0,7 & 0,42 \\
P22 & 1,0 & 0,6 & 0,6 & 0,36 \\
\hline & & & &
\end{tabular}

A vulnerabilidade média significa que $o$ aquífero é vulnerável a alguns contaminantes, mas somente quando continuamente lançados ou lixiviados. Já a vulnerabilidade alta significa que o aquífero é vulnerável a muitos contaminantes, exceto os que são fortemente adsorvidos ou rapidamente transformados (FOSTER et al. 2006).

MOURA et al. (2016) realizou estudo da vulnerabilidade aquífera na zona costeira do estado do Ceará, e considerou o Sistema Aquífero Dunas como o mais vulnerável, em comparação aos sistemas aquíferos Barreiras e Cristalino, tanto pelo método DRASTIC quanto pelo método GOD. Os resultados são semelhantes aos apresentados no presente estudo, no qual o principal fator de variação para a vulnerabilidade GOD é o nível estático.

A partir da avaliação do mapa de densidade de fossas (Figura 6) foram identificadas as áreas que apresentam diferentes graus de potencial à contaminação. A maior densidade ocorre nas porções centrais da área de estudo e, portanto, nestes 


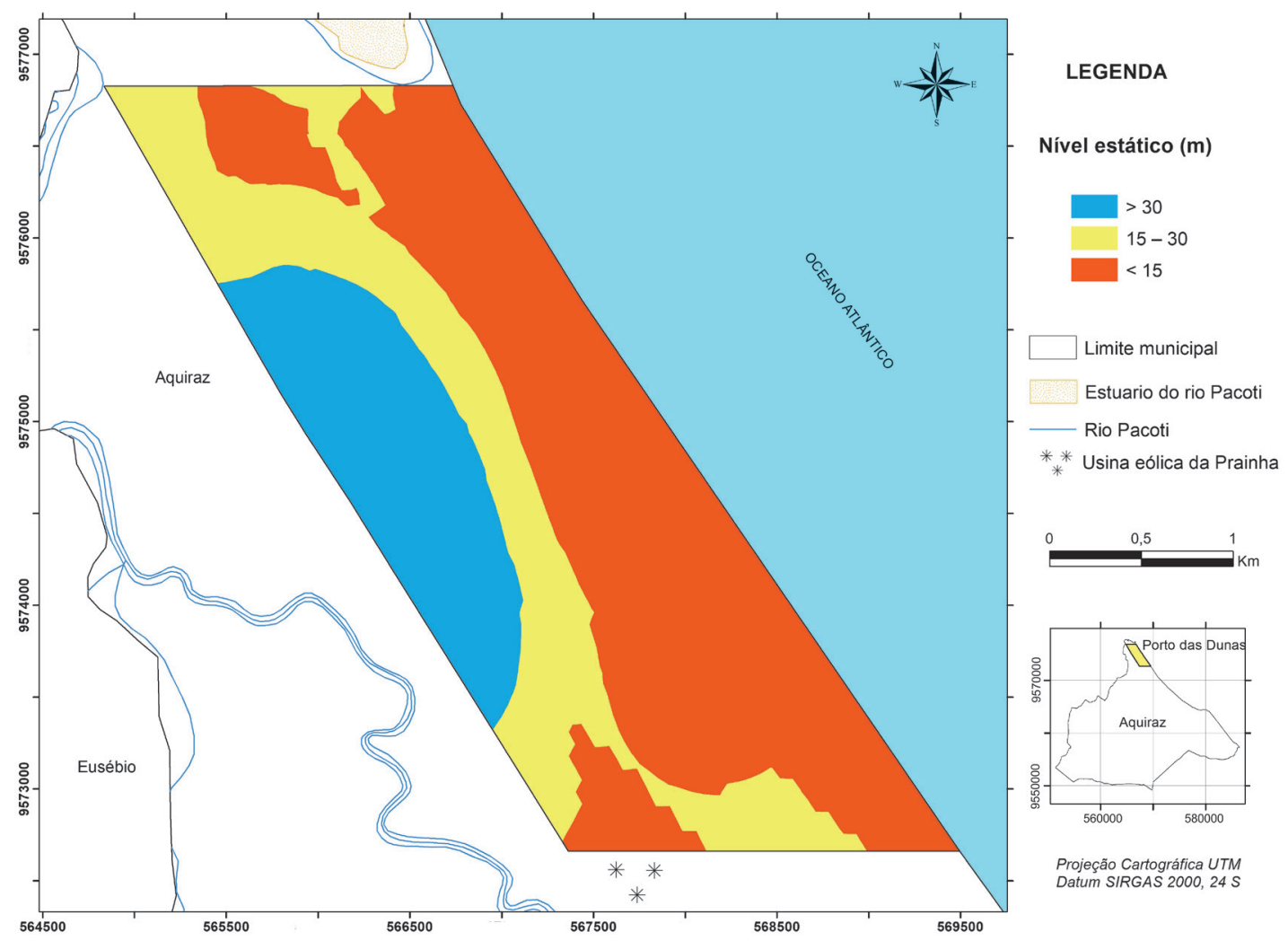

FIGURA 4 - Profundidade do nível estático no Sistema Aquífero Dunas.

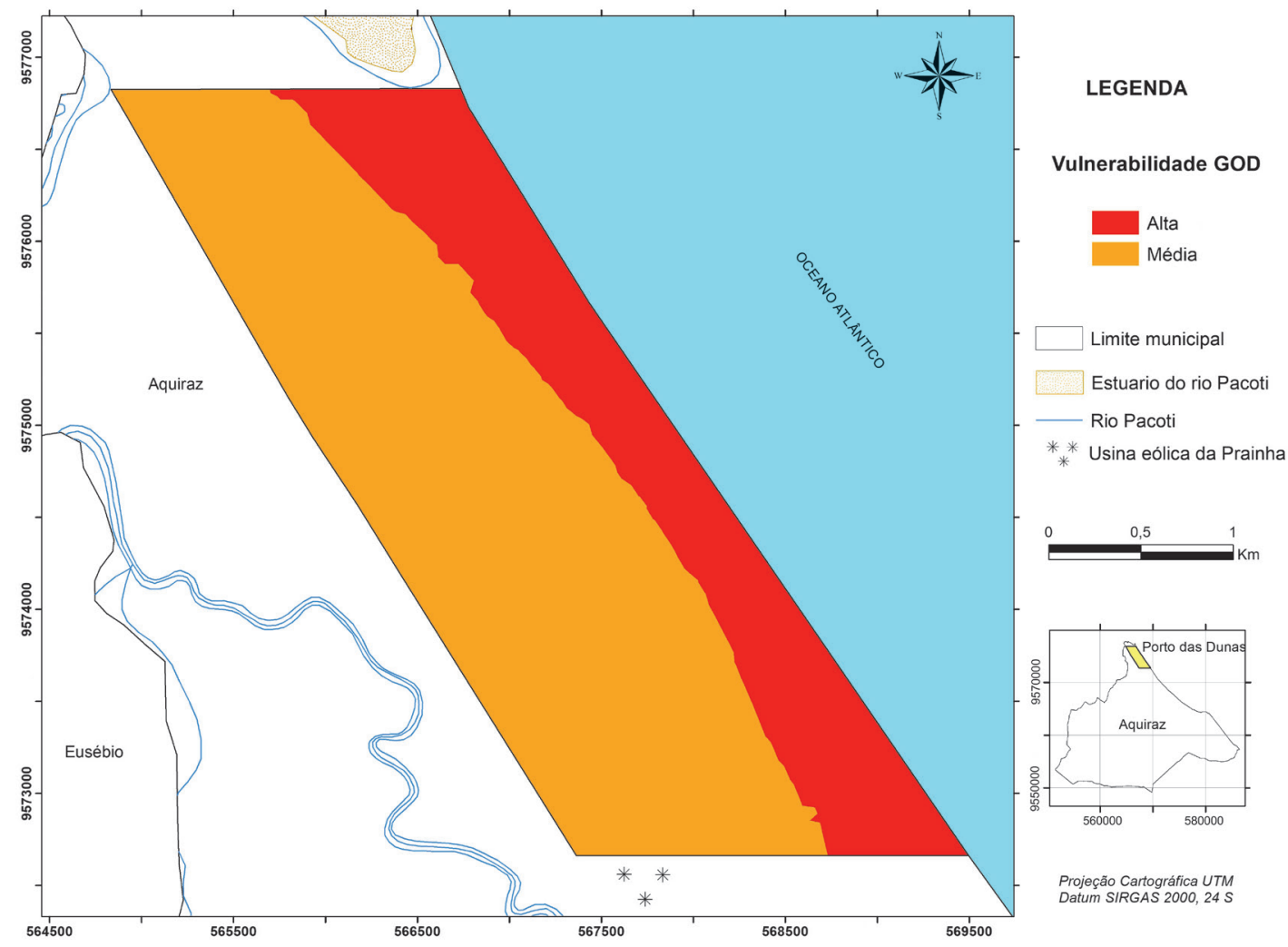

FIGURA 5 - Vulnerabilidade Natural GOD para o Sistema Aquífero Dunas. 
setores, o potencial à contaminação é mais elevado em comparação com os setores periféricos que apresentam menor densidade de fossas. Foram definidas três classes correspondentes aos seguintes valores de densidade, de acordo com a aplicação do estimador Kernel: densidade baixa $(<14,8)$, média $(14,8-82,2)$ e alta $(>82,2)$. Essa classificação foi definida de forma a atender o critério zonal para definição de área de maior perigo a contaminação. Visto que, em zonas de alta densidade de fossas a forma da contaminação passa a ser difusa à medida que diferentes plumas de contaminantes se encontram no meio subterrâneo (MITCHELL 2005).

Quanto ao perigo de contaminação, o cruzamento dos dados de vulnerabilidade e de densidade de fossas revelou que as áreas de maior perigo de contaminação são aquelas que apresentam alta densidade de fossas associadas às zonas de vulnerabilidade média e alta, bem como, áreas com média densidade de fossas associadas às zonas de alta vulnerabilidade (Figura 7).

MELO \& FIGUEREDO (1990) colocam que o principal fator de contaminação em áreas sem rede coletora de saneamento é, justamente, a proximidade das fossas com o poço de coleta, sejam poços tubulares ou manuais que captam o Sistema Aquífero Dunas. PEIXOTO (2016) em estudo feito em uma sub-bacia urbana na cidade de Fortaleza, considera que a densidade de fossas sépticas e rudimentares é o principal fator de contaminação por nitrato nas águas subterrâneas.

\section{CONCLUSÕES}

$\mathrm{Na}$ área de estudo o sistema aquífero Dunas, de acordo com a metodologia GOD, apresenta vulnerabilidade variando de média a alta. A vulnerabilidade média ocorre em grande parte da área de estudo e a alta na porção leste, próxima à linha de costa.

A variação da vulnerabilidade está relacionada à profundidade do nível estático, parâmetro $\mathrm{D}$, tendo em vista que devido as características do sistema aquífero, os parâmetros $\mathrm{G}$ e $\mathrm{O}$ foram os mesmos para todos os pontos. Com isso, o que se observa é que a vulnerabilidade é maior em zonas com nível estático menos profundo, devido à maior



FIGURA 6 - Densidade de fossas no Porto das Dunas, Aquiraz-CE. 


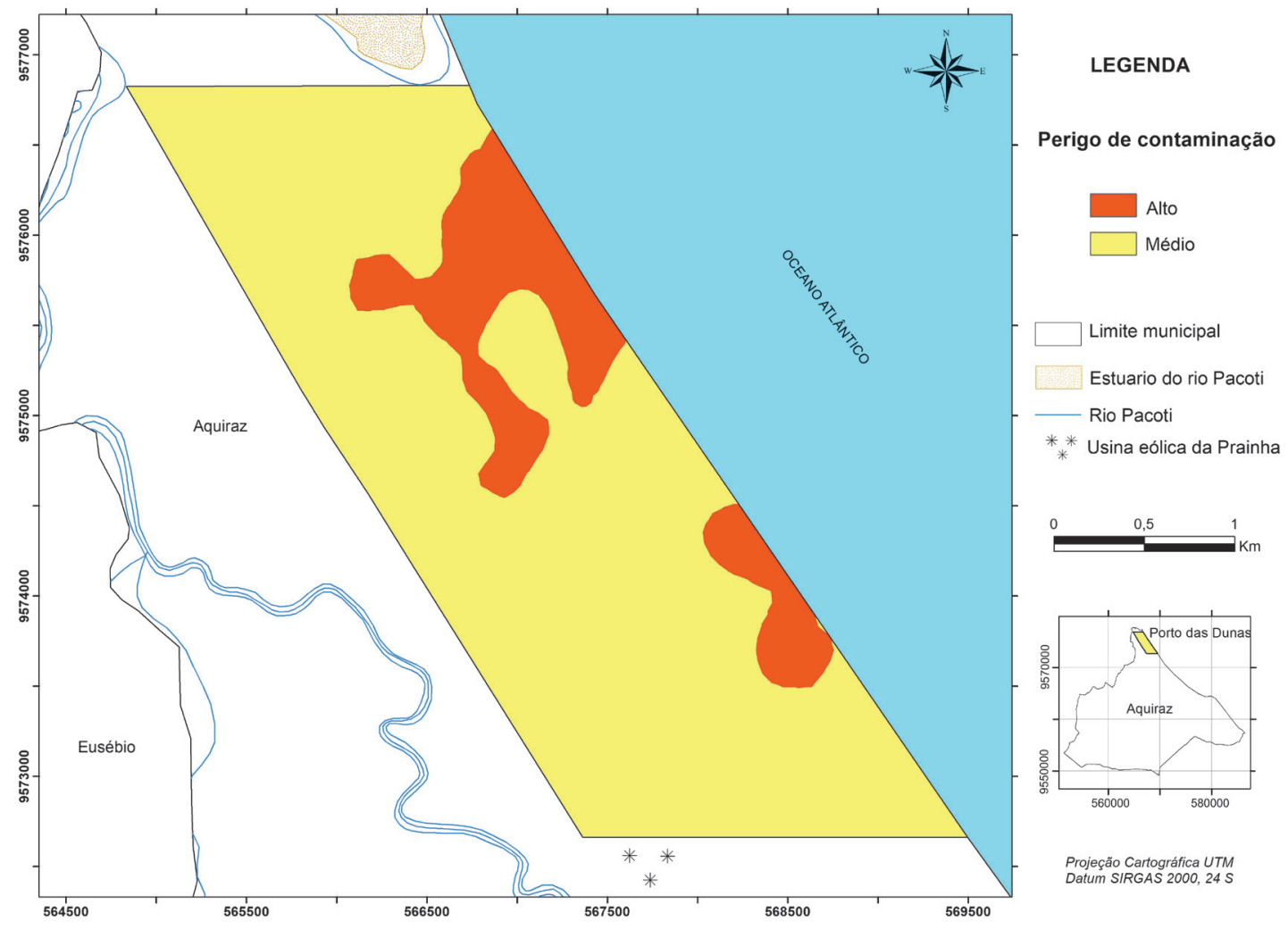

FIGURA 7 - Perigo de contaminação das águas subterrâneas no Porto das Dunas, Aquiraz-CE

facilidade de acesso de contaminantes à zona saturada do aquífero.

O grande número de fossas existente na região, decorrente da ausência de saneamento básico, resulta em aplicação constante e elevada de carga contaminante no subsolo. Por meio da análise da distribuição da densidade de fossas na área, associada aos índices vulnerabilidade do sistema aquífero Dunas, foram identificadas as áreas com maior perigo de contaminação, que se encontram nas zonas central e costeira da área de estudo.

Faz-se necessária a instalação de rede de abastecimento de água e esgoto em toda a área, bem como a manutenção periódica das fossas existentes, tendo em vista que a coexistência de poços domésticos para abastecimento e fossas em áreas de média a alta vulnerabilidade representa um risco à saúde da população.

Espera-se que os resultados apresentados neste trabalho possam servir de subsídios para a gestão dos recursos hídricos subterrâneos da região, bem como para o desenvolvimento de políticas públicas de uso e ocupação do solo que visem a proteção desses recursos.

\section{AGRADECIMENTOS}

Aos relatores anônimos da Revista do Instituto Geológico.

\section{REFERÊNCIAS BIBLIOGRÁFICAS}

ALLER, L.; BENNET, T.; LEHR, J.H.; PETTY, R.J. 1987. DRASTIC: A Standardized System for Evaluating Groundwater Pollution Using Hydrogeologic Settings. U.S. Environmental Protection Agency, 163 p. (EPA/600/287/035).

BOUFEKANE, A.; SAIGHI, O. 2013. Assesment of groundwater pollution by nitrates using intrinsic vulnerability methods: a case study of the Nil valley groundwater (JIjel, North-East Algeria). African Journal of Environment Science and Technology, 7(10): 949-960.

BRANDÃO, R.L. 1995. Sistema de Informações para Gestão e Administração Territorial da Região Metropolitana de Fortaleza - Proje- 
to SINFOR: Diagnóstico Geoambiental e os Principais Problemas de Ocupação do Meio Físico da Região Metropolitana de Fortaleza. CPRM, Fortaleza, $1^{\mathrm{a}}$ edição, 105 p. (Série Ordenamento Territorial, 1).

BRASIL. 2003. Resolução CONAMA N ${ }^{\circ} 341$, de 25 de setembro de 2003. Dispõe sobre critérios para a caracterização de atividades ou empreendimentos turísticos sustentáveis como de interesse social para fins de ocupação de dunas originalmente desprovidas de vegetação na Zona Costeira. Brasília, DF, 03 nov. 2003, 3 p.

CÂMARA, C.F.; LOURENÇO, R.M.; FARIAS, J.F.; SILVA, E.V. 2010. Estudo geoambiental do litoral da Prainha e do Porto das Dunas/ Aquiraz-CE: Propostas para o planejamento ambiental. In: Universidade de Coimbra, SEMINÁRIO LATINO AMERICANO DE GEOGRAFIA FÍSICA, 6, SEMINÁRIO IBERO AMERICANO DE GEOGRAFIA FÍSICA, 2, Coimbra, Livro de Atas Digital.

CAVALCANTE, I.N. 1998. Fundamentos Hidrogeológicos para a Gestão Integrada de Recursos Hídricos na Região Metropolitana de Fortaleza, Estado do Ceará. Instituto de Geociências, Universidade de São Paulo, São Paulo, Tese de Doutoramento, 164 p.

CAVALCANTE, J.C.; VASCONCELOS, A.M.; MEDEIROS, M.F.; PAIVA, I.P.; GOMES, F.E.M.; CAVALVANTE, S.N.; CAVALCANTE, J.E.; MELO, A.C.R.; DUARTE NETO, V.B.; BENEVIDES, H.C. 2003. Mapa Geológico do Estado do Ceará, Escala 1:500.000, CPRM, Fortaleza.

CPRM - SERVIÇO GEOLÓGICO DO BRASIL. 2013. Sistema de Informações de Águas Subterrâneas (SIAGAS). Disponível em http:// siagasweb.cprm.gov.br/layout/ pesquisa complexa.php. Acessado em 17 jun. 2013.

CUTRIM, A.O.; CAMPOS, J.E.G. 2010. Avaliação da Vulnerabilidade e Perigo à Contaminação do Aquífero Furnas na Cidade de Rondonópolis com a Aplicação dos métodos GOD e POSH. Revista Geociências, 29(3): 401-411.

FOSTER, S.; HIRATA, R.C.A. 1988. Groundwater pollution risk evaluation: the methodology using available data. CEPIS/PAHO/WHO, Lima, $78 \mathrm{p}$.

FOSTER, S.; HIRATA, R.; GOMES, D.; D'ELIA, M.; PARIS, M. 2006. Groundwater Quality Protection: A Guide for Water Service Companies, Municipal Authorities and Environment Agencies. World Bank, GWMATE, Washington, $104 \mathrm{p}$.

FREIRE, G.S.S. 1989. Etude Hydrologique et Sedimentologique de L'Estuaire du Rio Pacoti (Fortaleza - Ceará - Brésil). Faculté des Sciences et des Techniques, Université de Nantes, Thèse (Doctorat en Sciences de La Terre), $229 \mathrm{p}$.

HIRATA, C.A.R.; FERREIRA, L.M.R. 2001. Os Aquíferos da Bacia Hidrográfica do Alto Tietê: Disponibilidade Hídrica e Vulnerabilidade à Poluição. Revista Brasileira de Geociências, 31: 43-50.

IPECE - INSTITUTO DE PESQUISA E ESTRATÉGIA ECONÔMICA DO CEARÁ. 2012. Perfil Básico Municipal. Disponível em: http://www.ipece.ce.gov.br/publicacoes/perfil basico/pbm-2012/Aquiraz.pdf. Acessado em 14 mar. 2013.

KUMAR, P.;BANSOD, B.K.S.; DEBNATH, S.; THAKUR, P.K.; GHANSHYAM, C. 2015. Index-based groundwater vulnerability mapping models using hydrogeological settings: A critical evaluation. Environmental Impact Assessment Review, 51: 38-49.

LOPES, J.L.S.; CESTARO, L.A.; KELTING, F.M.S. 2012. Zoneamento ambiental como instrumento de uso e ocupação do solo do município de Aquiraz-CE. Boletim Goiano de Geografia, 32(1): 93-104.

MELO, J.G.; FIGUEREDO, E.M. 1990. Comportamento hidráulico e vulnerabilidade do sistema aquífero Dunas/ Barreiras à poluição na área de Natal (RN). Águas Subterrâneas, 13(1): 98-110.

MITCHELL, G. 2005. Mapping hazard from urban non-poit pollution: a screening model to support sustainable urban dranage planning. Jornal of Environmental Management, 74: 1-9.

MOURA, P.; SABADIA, J.A.B.; CAVALCANTE, I.N. 2016. Mapeamento de vulnerabilidade 
dos aquíferos Dunas, Barreiras e Fissural na porção norte do complexo industrial e portuário do Pecém, estado do Ceará. Revista Geociências, 35(1): 77-89.

PEIXOTO, F.S. 2016. Efeitos do uso e ocupação do solo nas águas subterrâneas a sudeste do município de Fortaleza - Ceará. Centro de Ciências, Universidade Federal do Ceará, Fortaleza, Dissertação de Mestrado, 130 p.

PEREIRA JÚNIOR, L.C.; SOARES, H.L.T.; CASTRO, S.S. 2015. Vulnerabilidade natural e risco de contaminação do aquífero Bauru no município de Rio Verde - GO. Águas Subterrâneas, 29(2): 129-145.

PONTES, C.R.C.; LASTORIA, G.; PARANHOS FILHO, A.C.; GABAS, S.G.; OLIVEIRA, P.T.S. 2009. Determinação da vulnerabili- dade do aquífero basáltico no campus da UFMS, em Campo Grande - MS. Águas Subterrâneas, 23(1): 105-120.

REGINATO, P.A.R.; AHLERT, S. 2013. Vulnerabilidade do sistema aquífero Serra Geral na região nordeste do Estado do Rio Grande do Sul. Águas Subterrâneas, 27(2): 32-46.

ROSENBERGER, M.; VARNIER, C.; IRITANI, M.A.; FERREIRA, L.M.R.; ODA, G.H.; VIOTTI, M. 2013. Vulnerabilidade natural à contaminação do sistema aquífero Bauru na área urbana do município de Bauru. Revista do Instituto Geológico, São Paulo, 34(2): 51-67.

SILVERMAN, B.W. 1986. Density Estimation for Statistics and Data Analysis. Chapman and Hall, Nova York.

Endereço dos autores:

Karen Vendramini de Araújo, Itabaraci Nazareno Cavalcante, Rafael Mota de Oliveira, Filipe da Silva Peixoto, Inácio Ocinaí de Lima Neto - Departamento de Geologia, Centro de Ciências, Universidade Federal do Ceará, Av. Humberto Monte, S/N, Bloco 912/913, Pici, CEP 60020-181, Fortaleza/CE. E-mails:karenvendramini@yahoo.com.br,itabaracicavalcante@gmail.com,rafaelmota20@yahoo.com.br, fpeixoto10ufc@gmail.com, inacioneto@ufc.br

Artigo submetido em 17 de agosto de 2017, aceito em de 28 de dezembro de 2017. 